\title{
Emotion affects action: Midcingulate cortex as a pivotal node of interaction between negative emotion and motor signals
}

\author{
Mirtes Garcia Pereira, Letícia de Oliveira, and Fátima Smith Erthal \\ Federal Fluminense University, Rio de Janeiro, Brazil \\ Mateus Joffily, Izabela F. Mocaiber, and Eliane Volchan \\ Federal University of Rio de Janeiro, Rio de Janeiro, Brazil \\ AND \\ Luiz Pessoa \\ Indiana University, Bloomington, Indiana
}

\begin{abstract}
Affective pictures drive the activity of brain networks and impact behavior. We showed previously that viewing unpleasant pictures interfered in the performance of a basic nonemotional visual detection task. In the present study, we employed functional magnetic resonance imaging to test the hypothesis that behavioral interference may result from the interaction between negatively valenced and motor-related signals in the brain. As in our previous study (Pereira et al., 2006), participants performed a simple target detection task that followed the presentation of unpleasant or neutral pictures. Our results revealed that an unpleasant emotional context modulated evoked responses in several regions engaged by the simple target detection task. In particular, the midcingulate cortex was recruited when participants performed target detection trials during the unpleasant context, and signal responses in this region closely mirrored the pattern of behavioral interference (as revealed via reaction time). Our findings suggest that the midcingulate cortex may be an important site for the interaction between negatively valenced signals and motor signals in the brain and that it may be involved in the implementation of defensive responses, such as freezing.
\end{abstract}

There is a large body of studies demonstrating that emotion impacts perception and behavior. On the one hand, beneficial effects have been reported, including findings that threat stimuli enhance contrast sensitivity (Phelps, Ling, \& Carrasco, 2006), low spatial-frequency sensitivity (Bocanegra \& Zeelenberg, 2009), and search efficiency for task-relevant objects (Becker, 2009). On the other hand, the deleterious effects of emotional content are also well documented. In particular, unpleasant stimuli compete effectively for visual processing resources and often impair behavioral performance when their processing is irrelevant for the task at hand. For example, determining the orientation of a target visual stimulus was slower following emotional pictures (Hartikainen, Ogawa, \& Knight, 2000), and the presence of a central unpleasant picture increased response times (RTs) when participants discriminated peripheral target letters (Tipples \& Sharma, 2000) or the orientation of bars (Erthal et al., 2005). These effects of emotional stimuli on performance are commonly thought to be mediated by attention (Pessoa, Kastner, \& Ungerleider, 2002; Vuilleumier, 2005). In particular, it is believed that interference is due to the initial consumption of resources by emotional items or, possibly, to an increased difficulty in disengaging from emotional information (Bradley, Cuthbert, \& Lang, 1996; Koster, Crombez, Verschuere, \& De Houwer, 2004). This attention-like competitive advantage of emotional stimuli appears to be mediated by the amygdala (Anderson \& Phelps, 2001).

Viewing unpleasant stimuli also generates defensive reactions (Azevedo et al., 2005; Bradley, Codispoti, Cuthbert, \& Lang, 2001) and prepares participants for action (Hajcak et al., 2007). Darwin (1872) argued that emotions are adaptive insofar as they prompt actions that are beneficial to the organism. Contemporary theories of emotion are based on the belief that, in order to survive, animals should be capable of identifying threat signals that allow them to avoid threats to their body envelope. From this perspective, it would be expected that the processing of emotional items would modulate signals in motor-related areas. Indeed, accumulating evidence (e.g., Graziano $\&$ Cooke, 2006) has demonstrated the involvement of motor-related cortical areas during threatening contexts in the monkey. This notion is also consistent with studies that 
have reported increased motor cortex excitability during emotional processing in humans, as assessed via the combination of electromyography and transcranial magnetic stimulation (Baumgartner, Willi, \& Jäncke, 2007; Hajcak et al., 2007; Oliveri et al., 2003). Other neuroimaging studies have reported that activity in primary motor cortex and putamen is robustly modulated during experimentally induced states of fear (Butler et al., 2007; Phelps et al., 2001), consistent with the idea that aversive contexts engage motor circuits. In a recent neuroimaging study (Morrison, Peelen, \& Downing, 2007), activity in cingulate areas, such as midcingulate cortex, increased during unpleasant trials, and the activation depended on whether or not the participant made an overt motor response to the event. The findings concerning the midcingulate cortex are of particular interest because these medial regions have been characterized as medial premotor areas (Koski \& Paus, 2000) and are known to project topographically to primary motor cortex, supplementary motor areas, and putamen, in addition to having direct connections to the spinal cord (Morecraft, Louie, Schroeder, \& Avramov, 1997). Notably, the midcingulate cortex receives widespread inputs, both directly and indirectly, from emotionrelated brain regions (Paus, 2001) and may be a pivotal node of emotion and motor integration (Morecraft \& Van Hoesen, 1998).

As previously stated, the impact of affective stimuli on attention has been explored as an important component underlying emotional modulation (Vuilleumier, 2005). However, the potential role of interactions between affective processing and motor processes in behavioral interference has been less explored. In a recent study (Pereira et al., 2006), we reported a long-lasting interference effect produced by unpleasant picture viewing. Specifically, participants exhibited a marked slowing down of RTs when performing a simple target detection task during unpleasant blocks, as compared with neutral blocks. Because sustained behavioral interference was observed only during the blocked presentation of stimuli, we suggested that viewing negative pictures may have caused the induction of an emotional state linked to the activation of defensive responses. We further suggested that the triggering of these defensive, "freezing"-like motor patterns might have contributed to the sustained interference observed in our behavioral study.

The goal of the present study was to probe the neural underpinnings of sustained behavioral interference. Specifically, we were interested in probing how behavioral interference during unpleasant picture viewing was related to motor-related evoked responses. We hypothesized that generating a motor response to a neutral target in an emotional context was associated with modulation of activity in a network of brain regions involved in motor execution. To address this question, we employed a paradigm analogous to the one that we used previously (Pereira et al., 2006; Pereira et al., 2004), in which sustained behavioral interference was observed. We further reasoned that, if the interaction of affective and motor-related signals underlies behavioral interference effects, evoked responses due to unpleasant picture viewing would be correlated with evoked brain responses during motor action. Finally, we anticipated that the slow-down in RTs during unpleasant blocks would be tied to responses in brain regions involved in the integration of emotion and motor signals, such as the cingulate cortex.

\section{METHOD}

\section{Participants}

Eleven right-handed male participants (18-32 years old, $M=$ 24.8) participated in the study. All had normal or corrected-tonormal vision, reported no psychiatric or neurologic problems, and were not under medication with central nervous system action. The experiment was approved by the IRB of the Federal University of Rio de Janeiro, and the participants gave written informed consent.

\section{Stimuli}

Sixty pictures (30 neutral and 30 unpleasant) were employed. Twenty-two pictures (6 neutral and 16 unpleasant) were obtained from the International Affective Picture System (IAPS), and the remaining pictures were either obtained from the World-Wide Web or photographed by the authors (those additional images were obtained because those available in the IAPS set were not sufficient). All of the pictures were of the same size $(640 \times 480$ pixels $)$. The neutral pictures consisted of photographs of people, and the unpleasant pictures consisted of photographs of mutilated bodies. We attempted to match unpleasant and neutral stimuli in terms of both color content and complexity (e.g., number of faces, number of body parts, etc.). Following the protocol developed by Lang, Bradley, and Cuthbert (1997), the pictures were rated on a scale of 1-9 in terms of valence (from negative to positive) and arousal (from low to high) by a separate group of participants $(N=40)$ using the paper-and-pencil version of the Self-Assessment Manikin (Bradley \& Lang, 1994). Overall, the pictures in the neutral category had a mean valence rating of $4.9(S D=0.3)$ and a mean arousal rating of $3.3(S D=0.6)$. The pictures in the unpleasant category had a mean valence rating of $2.2(S D=0.4)$ and mean arousal rating of $6.3(S D=0.6)$. An additional set of 6 neutral pictures of household objects from the IAPS data set was selected for use during a practice block.

\section{Design and Procedure}

Visual stimuli were displayed by means of magnetically shielded LCD goggles (Resonance Technologies, Inc., Northridge, CA). The experimental session was divided into two runs. Each run consisted of two detection-only blocks: one unpleasant block and one neutral block. Unpleasant and neutral blocks lasted $225 \mathrm{sec}$ each, and detection-only blocks lasted $60 \mathrm{sec}$. All blocks were followed by a $12-\mathrm{sec}$ fixation baseline. During the unpleasant and neutral blocks, each picture was presented for $3 \mathrm{sec}$ and was immediately followed by a sequence of eight target detection trials (Figure 1). Picturecontaining blocks consisted of a single type of stimulus, unpleasant or neutral, and block order was counterbalanced across participants. Within unpleasant and neutral blocks, the order of presentation of the 15 pictures was randomized for each participant. Participants were instructed to attend to each picture for as long as it was displayed. Each target detection trial consisted of a fixation spot presented at the center of the screen 500-700 msec prior to target onset. The target was a small annulus that appeared around the fixation spot; both remained on until the participant responded (or until 1,500 msec had elapsed). Both the fixation spot and the target were presented in white on a black background. Participants were required to press a button with the right index finger as quickly as possible after target onset. A new target detection trial began $500 \mathrm{msec}$ after the participant's response. After eight target detection trials, a new picture appeared on the screen. Target detection trials with RTs shorter than $100 \mathrm{msec}$ or longer than $1,000 \mathrm{msec}$ were considered errors and were discarded from the RT analysis. Detection-only blocks were identical to picture-containing blocks, except that a 3 -sec black screen 

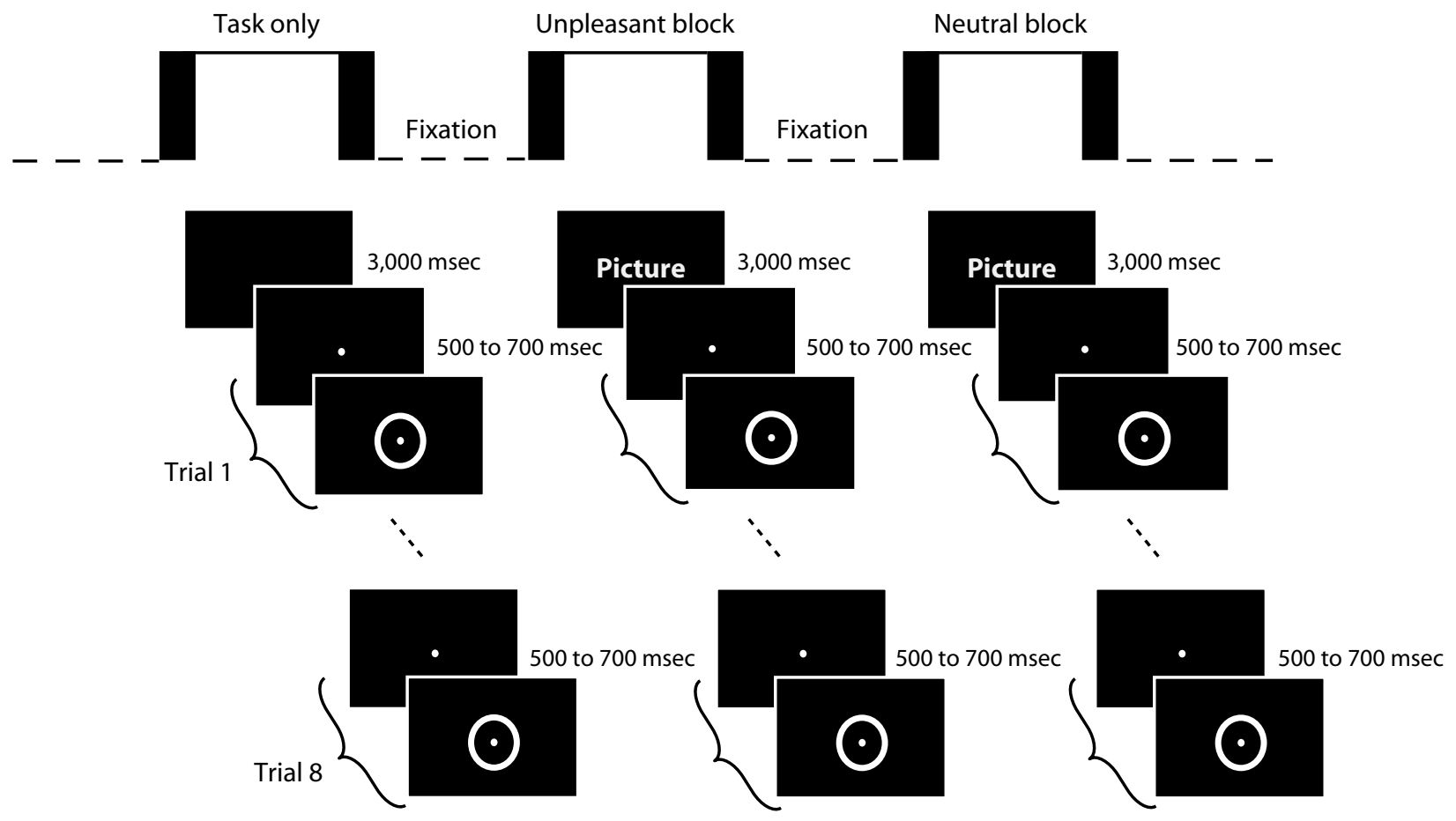

Figure 1. Experimental design. Each experimental run consisted of two detection-only blocks, one unpleasant block, and one neutral block. During the unpleasant and neutral blocks, pictures were presented for 3 sec and were immediately followed by a sequence of eight target detection trials. Participants were instructed to attend to each picture for as long as it was displayed and to press a button with the right index finger as quickly as possible after target onset. After eight target detection trials, a new picture appeared on the screen. In the detection-only blocks, a 3-sec black screen preceded the sequence of target detection trials. Stimuli are not drawn to scale.

preceded the sequence of target detection trials. At the beginning of the session, during anatomical scanning, participants performed a practice block, which was similar in structure to the neutral block, except that all images involved photographs of neutral objects, such as tools and furniture, instead of people.

\section{Image Acquisition}

Functional MRI data were collected using a 1.5T MRI scanner (Magnetom Vision Plus, Siemens, Erlangen, Germany). Functional images were acquired using a gradient-echo echo-planar imaging sequence $\left(\mathrm{T}_{\mathrm{R}}=3 \mathrm{sec} ; \mathrm{T}_{\mathrm{E}}=60 \mathrm{msec} ; \mathrm{FOV}=240\right.$; flip angle $=90^{\circ}$; $64 \times 64$ matrix). Whole-brain coverage was obtained with 25 axial slices (thickness $=4 \mathrm{~mm}$; in-plane resolution $=3.75 \times 3.75 \mathrm{~mm}$ ). Echo-planar images were coregistered to a high-resolution structural T1-weighted image obtained during the same session $\left(\mathrm{T}_{\mathrm{R}} /\right.$ $\mathrm{T}_{\mathrm{E}}=9.7 / 4.0 \mathrm{msec}$; flip angle $=12^{\circ} ; 128$ sagittal slices; thickness $=$ $1.25 \mathrm{~mm} ; 256 \times 256$ matrix; FOV $=256 \mathrm{~mm}$ ). Head movements were restrained by foam padding. We acquired 234 functional volumes during each of two runs, each of which lasted approximately 12 min. Stimulus presentation was synchronized with MRI data acquisition.

\section{RT Data Analysis}

Two participants were excluded from RT analysis due to excessive errors $(>15 \%$; mean error rate $=1.3 \%)$. Because the resulting sample size $(N=9)$ was relatively small, to increase statistical power, we ran the exact same experiment with an additional sample (22 males, mean age $=24.8$ years) outside of the scanner. The RTs for successive Target Detection Trials 1 and 2, 3 and 4, 5 and 6, and 7 and 8 were averaged, resulting in four mean RTs (henceforth referred to as Target Detection Trials 1-4). Data from both groups were included in a repeated measures ANOVA with within-participants factors of valence (unpleasant, neutral) and target detection trial (1-4), and a between-participants factor of group (inside, outside). Note that the RTs from Trials $1-8$ were not averaged into a single value because we were interested in evaluating the time course of the interference effect - for example, whether it was sustained or not. We employed averaged trials to diminish the number of levels in the above ANOVA from eight to four (as done in our previous behavioral study; Pereira et al., 2006).

\section{fMRI Data Analysis}

The statistical parametric mapping software package (SPM2, Wellcome Department of Cognitive Neurology, London) was used for preprocessing and statistical analyses. The first three functional volumes of each run were removed to eliminate nonequilibrium effects of magnetization. The remaining images were corrected for head movement by realigning all the images to the first image via rigid body transformations. The images were then corrected for differences in slice acquisition time. For each participant, functional and structural images were coregistered. Structural data were normalized by matching them to the standardized MNI template, and the transformation parameters estimated in this step were applied to all functional images. Functional images were spatially smoothed with an 8-mm full width at half maximum Gaussian kernel prior to statistical analysis.

Data analysis was performed according to the general linear model framework, as implemented in SPM2 (Friston et al., 1994). Data obtained from the 11 participants were analyzed. The first (fixed) level involved determining regression coefficients of variables of interest, which modeled the effects of each experimental condition: detection only, viewing neutral pictures, viewing unpleasant pictures, detection after neutral pictures, and detection after unpleasant pictures. Before estimation via multiple regression, 
regressors of interest were convolved with a canonical hemodynamic response function. Because the target detection phase of picturecontaining blocks followed the pictures immediately, detectionrelated regressors modeled only Target Detection Trials 3-8 (thus excluding the first two target detection trials). We adopted this fairly conservative strategy to minimize any spillover from the pictureviewing phase into the detection phase, a notion that was supported by the absence of emotional modulation during the target detection phase in visual cortex (as indicated by the unpleasant-detection vs. neutral-detection contrast). Note that specific phases of picturecontaining blocks were contrasted (e.g., unpleasant viewing vs. neutral viewing, but not against detection-only blocks). The latter were contrasted against the baseline condition in order to delineate the basic circuit engaged by the target detection task.

Second-level group analyses were conducted by means of repeated measures ANOVAs and $t$ tests. Since random effects analyses may be fairly conservative in the context of fMRI data (Worsley et al., 2002), we employed a threshold of $p<.001$ (uncorrected), as is commonly employed in the literature.

Additional region-of-interest (ROI) analyses were also performed. In each case, a voxelwise contrast (e.g., detection only vs. baseline) was employed first to determine a set of candidate clusters, which were further interrogated via additional contrasts (e.g., unpleasant detection vs. neutral detection). The local peaks of the activation of the voxelwise contrasts were used as the center of a 10-mm-radius sphere around the peak voxel, and voxels within the sphere were further considered if they were statistically significant (a threshold of $p<.005$, uncorrected, was employed, except for the all-conditions vs. baseline contrast, which employed $p<.001$, uncorrected, given the strength of this contrast). Mean regression coefficients were then determined for each region. ROI construction and signal extraction were performed using the MarsBar toolbox for SPM2. Note that all of our ROI analyses avoided circularity, in that the statistical test at the ROI level was independent from the selection criterion used to determine the ROI (Kriegeskorte, Simmons, Bellgowan, \& Baker, 2009; Vul, Harris, Winkielman, \& Pashler, 2009).

Overall, three main types of inferential ROI analysis were performed. Here, we briefly describe them and the rationale for carrying them out. In the first, a set of ROIs defined via the detection-only versus baseline contrast was probed with the unpleasant-detection versus neutral-detection contrast (see Table 1 for a list of the ROIs). The objective of this analysis was to probe the basic circuit involved in target detection for modulations based on stimulus category (unpleasant vs. neutral).

In the second ROI analysis (see Figure 2), midcingulate cortex was identified via the all-conditions versus baseline contrast (note that the detection-only vs. baseline contrast does not engage the midcingulate cortex; an emotionally evocative stimulus appears to be necessary). The goal of this analysis was to investigate effects of both stimulus category and target detection trial, which was accom-

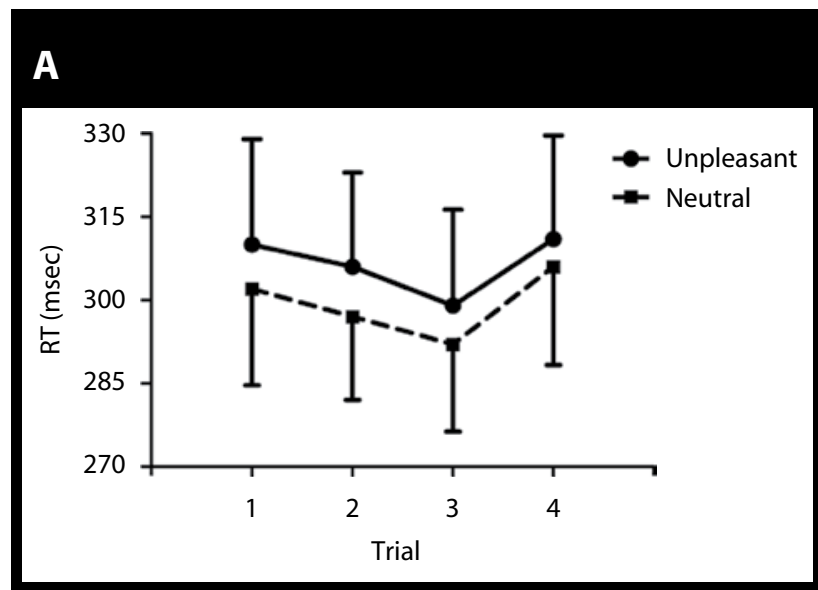

B

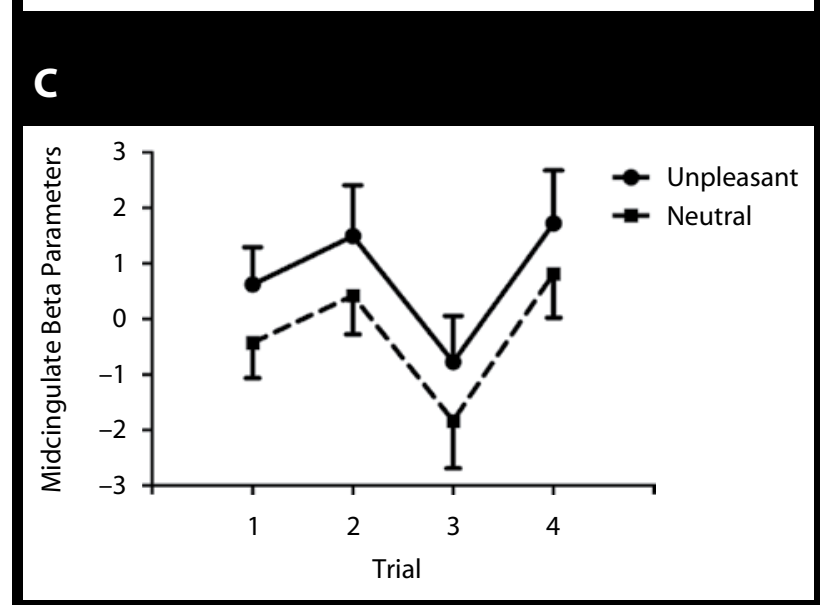

D

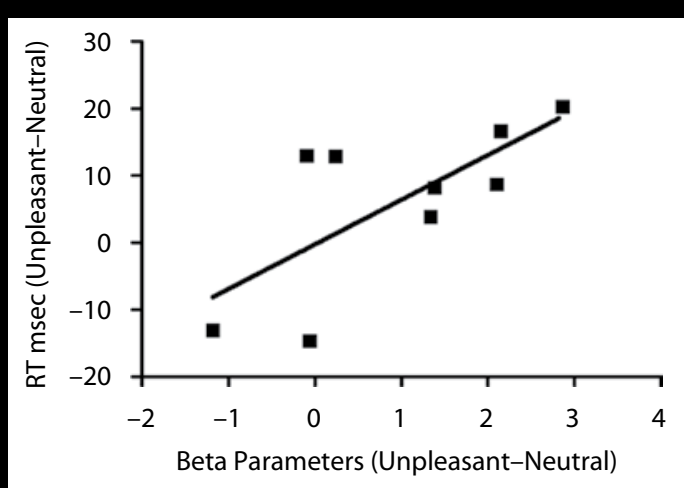

Figure 2. Midcingulate cortex responses and behavior. (A) Response time (RT) data are shown as a function of pooled target detection trial number and viewing condition. (B) Illustration of the midcingulate region of interest (ROI). (C) Midcingulate cortex ROI responses (MNI coordinates: $-8,12,46$ ) are shown as a function of pooled target detection trial number and viewing condition. (D) Scatterplot illustrating the linear relationship between behavioral and $\mathrm{fMRI}$ data. Error bars in $\mathrm{A}$ and $\mathrm{C}$ indicate the standard errors of the means. 


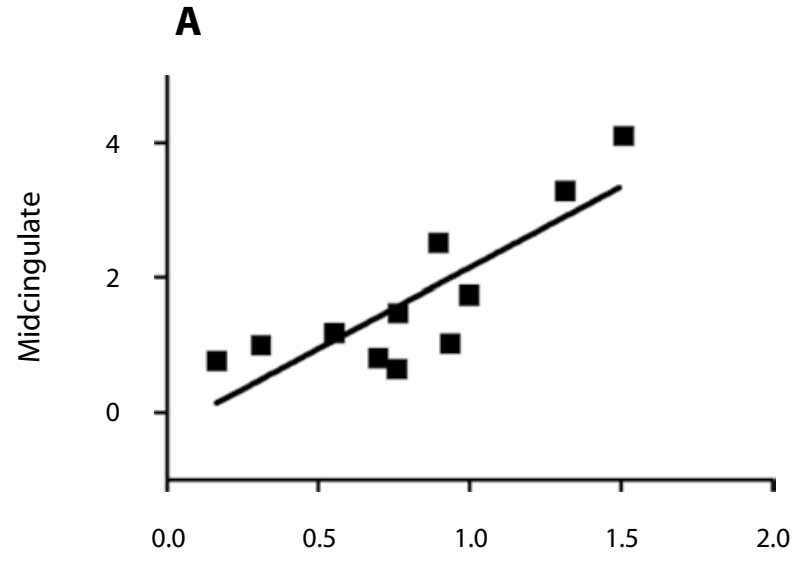

Middle Insula

B

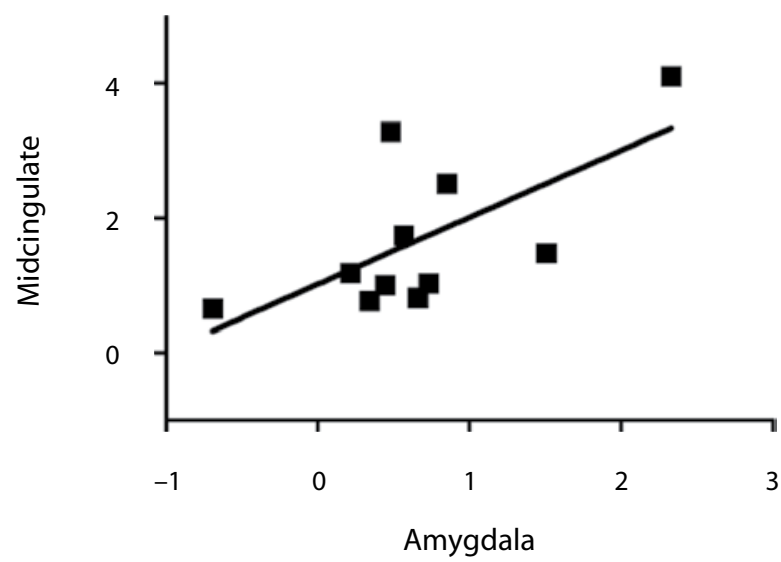

Figure 3. (A) Scatterplot illustrating the linear relationship between responses in the middle insula (unpleasant viewing vs. neutral viewing) and midcingulate cortex (detection unpleasant vs. neutral detection). (B) Scatterplot illustrating the linear relationship between responses in the amygdala (unpleasant viewing vs. neutral viewing) and midcingulate cortex (detection unpleasant vs. neutral detection).

plished via a 2 (valence: unpleasant, neutral) $\times 4$ (target detection trial: $1-4$ ) repeated measures ANOVA; as in the behavioral analysis, the latter factor was employed to probe the temporal unfolding of the interference effect.

In the third ROI analysis (see Figure 3), we focused on regions that exhibited increased responses to unpleasant viewing relative to those to neutral viewing and investigated how they were correlated with midcingulate signals. Accordingly, we tested the three regions differentially recruited by this contrast-namely, middle occipital gyrus, middle insula, and the amygdala. Signals from these regions were then correlated with responses observed in the midcingulate cortex. The latter was defined via the unpleasant-detection versus neutral-detection contrast because we were interested in relating midcingulate responses during detection to those in the structures above during stimulus viewing. In other words, we were interested in testing a link between responses during viewing and detection that would be consistent with a causal relationship (naturally, "consistent with" does not imply causation).

Finally, Figure 4 plots average fMRI responses in the middle occipital gyrus, middle insula, and midcingulate cortex (all regions were defined as described above for the third ROI analysis). The ac- tivity of voxels within an ROI was normalized ( $z$ score) with respect to the mean activity of each run, and trial averaging was used to generate the time courses shown. Our objective here was to illustrate how transient or sustained the responses were in these regions (no statistical inferences were performed).

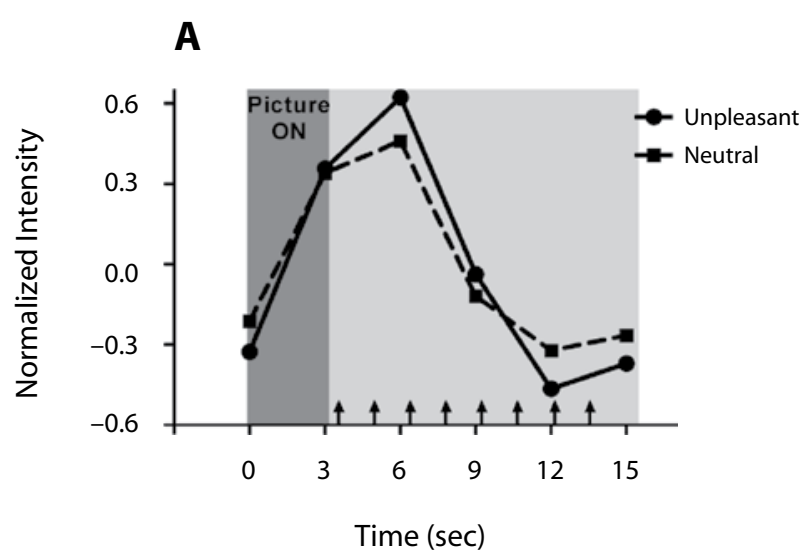

B

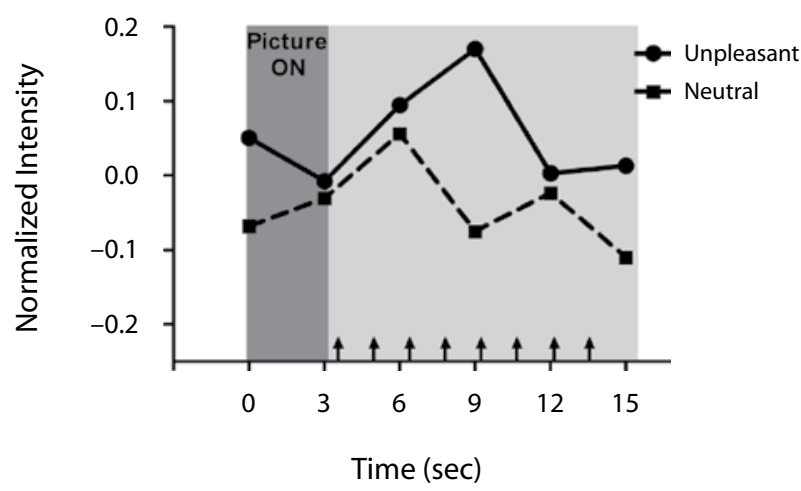

C

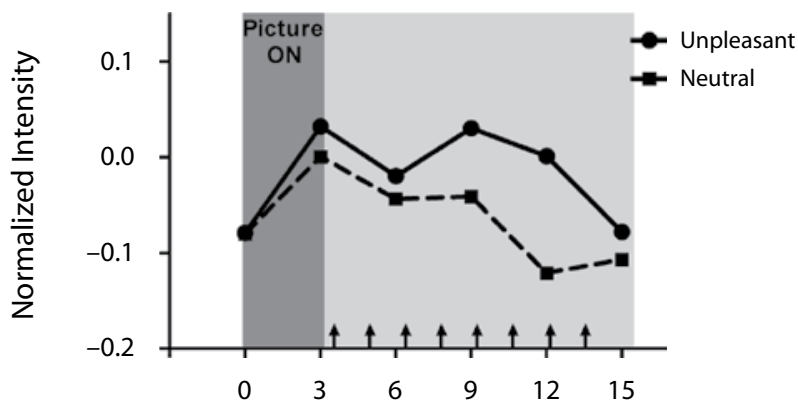

Time (sec)

Figure 4. Mean normalized signal during unpleasant and neutral blocks. The dark gray shaded area indicates the picture viewing phase, and each arrow in the light gray area approximately indicates the presentation of a detection target. Responses are shown for the (A) left occipital middle gyrus, (B) left middle insula, and $(C)$ left midcingulate cortex. Whereas the responses in visual cortex were fairly transient and driven by picture viewing, those in the middle insula and midcingulate cortex were more directly linked to the target detection phase. 


\section{RESULTS}

\section{Behavioral Performance}

RT data were analyzed according to a 2 (valence: unpleasant, neutral) $\times 4$ (target detection trial: $1-4) \times$ 2 (group: inside, outside) repeated measures ANOVA. Note that the factor target detection trial allowed us to evaluate the time course of the interference effect (e.g., transient vs. sustained). The analysis revealed a significant main effect of valence only $[F(1,29)=7.17, p=.01]$. In particular, no interactions involving valence were statistically significant [for valence $\times$ target detection trial, $F(3,87)=0.81, p=.5$; for valence $\times$ group, $F(1,29)=$ $0.12, p=.73$; for valence $\times$ target detection trial $\times$ group, $F(3,87)=1.1, p=.3]$. Post hoc comparisons revealed that all target detection trials performed during unpleasant blocks were significantly slower than those performed during neutral blocks (all $p \mathrm{~s}<.05$ ). This sustained effect over target detection trials is depicted in Figure 2A, where mean RTs during the unpleasant and neutral blocks are shown for the participants of the fMRI sample. This plot clearly illustrates the same general pattern of longlasting interference that we have reported in previous investigations (Pereira et al., 2006; Pereira et al., 2004). In fact, post hoc comparisons of the data from the fMRI sample $(n=9)$ revealed that the RTs of target detection trials performed during unpleasant blocks were slower than during neutral ones (all $p \mathrm{~s} \leq .05$, except for Target Detection Trial 4, for which $p=.29$ ).

\section{Brain Functional Activity}

Simple target detection task circuit. To identify the set of brain regions that was engaged by the target detection task, the detection-only condition was contrasted against the fixation baseline, which revealed increased responses in the left precentral gyrus, right middle frontal gyrus, left middle frontal gyrus, and, subcortically, bilateral putamen (Figure 5 and Table 1).

Brain areas activated by viewing mutilation pictures. To identify the set of brain regions engaged by mutilation stimuli, we contrasted unpleasant picture viewing against neutral picture viewing, which revealed enhanced activation in the occipital cortex and middle insula (Figure 6). Note that, whereas the responses in the former were relatively transient, those in the latter were less so (Figure 4; see the Method section). Given the large literature showing the involvement of the amygdala in similar conditions, a more lenient threshold for statistical significance was adopted ( $p<.05$, uncorrected), which revealed differential responses in the amygdala bilaterally (Figure 6C).

Emotional modulation of the circuit involved in target detection. The behavioral results revealed a longlasting slowdown of target detection when performed during the unpleasant condition. We thus interrogated motor-related regions identified via the detection-only versus baseline contrast for potential effects of emotional picture viewing. Specifically, a set of ROIs defined via the detection-only versus baseline contrast (see Table 1) was probed with the unpleasant-detection versus neutraldetection contrast, which revealed increased responses in the left precentral gyrus, left middle frontal gyrus, and bilateral putamen (Figure 5). This analysis thus revealed that most of the neural circuit engaged by the target detection task exhibited increased responses when target detection was performed after the viewing of unpleasant pictures.

Areas recruited during task performance in the unpleasant block. Were additional brain regions recruited during target detection in the unpleasant block? To answer this question, we conducted a whole-brain analysis contrasting the unpleasant-detection and neutraldetection conditions (Figure 7). Increased activation during the unpleasant-detection condition was observed in the inferior parietal gyrus, precentral gyrus, postcentral gyrus, midcingulate cortex, superior frontal gyrus, and putamen, all in the left hemisphere. Note that not all areas observed in the ROI analysis above were identified here because they did not reach the statistical threshold of the voxelwise analysis.

Signal modulation in the midcingulate cortex parallels behavioral interference. The recruitment of the midcingulate cortex when participants performed the target detection task during an aversive context was particularly interesting, considering that this area has been suggested as a critical node in the interaction between emotion and motor systems (Morecraft \& Van Hoesen, 1998; Morrison et al., 2007; Paus, 2001). To investigate whether signal responses in this region were related to the behavioral RT interference, we analyzed midcingulate fMRI data in a manner analogous to that done with the behavioral data - specifically, by considering responses for Average Target Detection Trials 1 through 4 (i.e., Original Trials 1 and 2, 3 and 4, 5 and 6, and 7 and 8 were averaged, producing Average Trials 1-4). A cluster of activation in the midcingulate cortex identified via the all-conditions versus baseline contrast was used to create an ROI for this region (peak voxel: $x=-8, y=12, z=46$; Figure 2). A 2 (valence: unpleasant, neutral) $\times 4$ (target detection trials: $1-4)$ repeated measures ANOVA revealed a statistically significant main effect of valence only $[F(1,10)=$ $4.87, p=.05]$. The estimated regression coefficients during the unpleasant and neutral conditions are illustrated in Figure 2, suggesting that midcingulate cortex responses paralleled the behavioral interference results. The relatively sustained increase in activity during the unpleasant block when participants were performing target detection trials is further illustrated in Figure 4C.

To further explore the relationship between midcingulate responses and behavioral interference, we tested whether participants with stronger evoked responses in the midcingulate ROI also exhibited the largest behavioral interference effects. Indeed, when differences in RT (unpleasant minus neutral) were regressed on regression coefficients (unpleasant minus neutral), a linear trend approached significance $(r=.62, p=.07)$ (Figure 2D).

Correlations between midinsula responses during picture viewing and midcingulate responses during target detection. Finally, we tested whether the emotional modulation observed in the midcingulate cortex during target detection performance was linked to responses evoked during the viewing of unpleasant stimuli. 

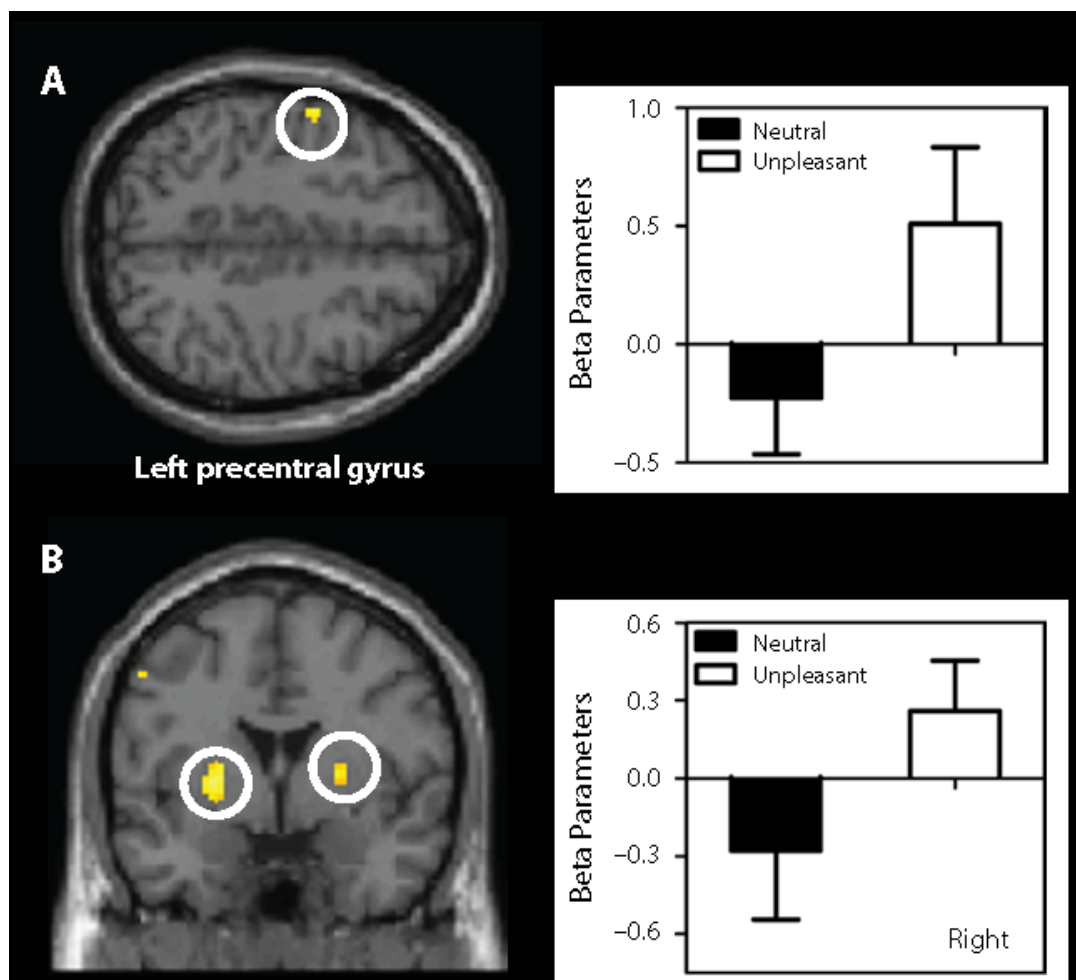

\section{Left and right putamen}
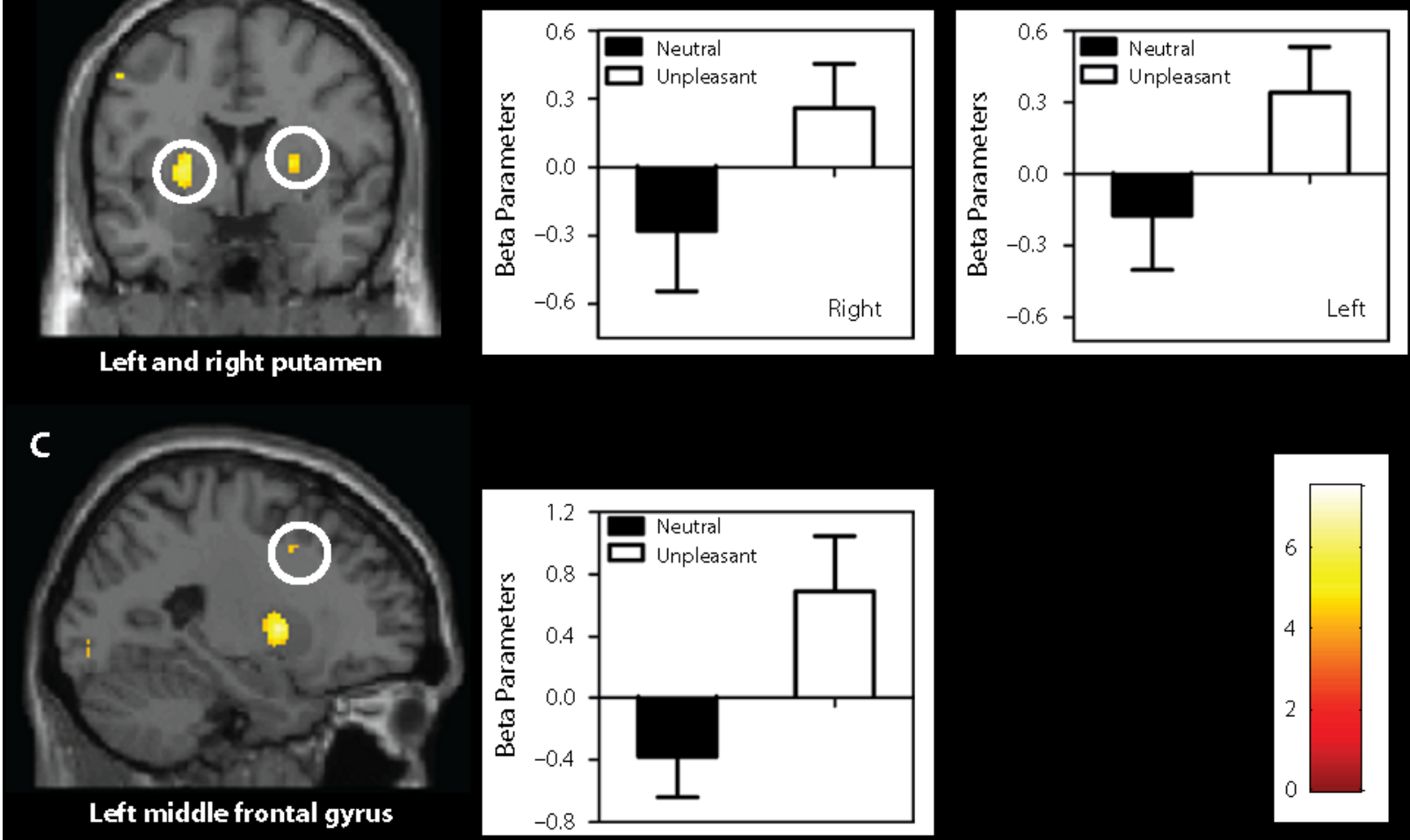

Figure 5. Voxelwise group maps on the left illustrate the results of the detection-only versus baseline contrast. These regions were further interrogated on the basis of the unpleasant-detection versus neutral-detection contrast, as shown in the bar plots on the right. Error bars indicate the standard errors of the means. The gradient scale represents $z$ values.

We reasoned that an area that responded more strongly during unpleasant picture viewing would possibly convey the information about the aversiveness of the context to the midcingulate cortex. To probe this, we conducted a correlation analysis between the clusters activated by the unpleasant-viewing versus neutral-viewing contrast (see Table 1) and the cluster of evoked activity in the midcingulate cortex, as defined from the unpleasant-detection versus neutral-detection contrast. Significant correlations were observed between the middle insula and the left midcingulate cortex $(r=.83, p<.005)$ and between the left amygdala and the left midcingulate cortex $(r=$ $.67, p<.05$ ) (Figure 3). No significant correlation was observed between activation of the early visual cortex, which was also more strongly engaged during unpleasant viewing, and that of the midcingulate cortex $(r<.2)$.

\section{DISCUSSION}

The objective of the present study was to investigate interactions between affective and motor processes. We were interested in the potential emotional modulation of output systems, so we chose a simple detection task not involving additional processing stages, such as those involving discrimination and/or response choice. The behavioral data replicated the findings of our previous study (Pereira et al., 2006) and revealed a sustained interference effect when a simple target detection task was executed 
Table 1

Brain Activations

\begin{tabular}{|c|c|c|c|c|c|c|}
\hline \multirow[b]{2}{*}{ Brain Region } & & \multicolumn{3}{|c|}{ MNI Coordinates } & \multirow{2}{*}{$\begin{array}{c}\text { Cluster } \\
\text { Size }\end{array}$} & \multirow[b]{2}{*}{$t$ Value } \\
\hline & & $x$ & $y$ & $z$ & & \\
\hline \multicolumn{7}{|l|}{ Detection Only Versus Baseline } \\
\hline Lingual gyrus & $\mathrm{R}$ & 24 & -88 & -10 & 58 & 6.25 \\
\hline Middle occipital gyrus & $\mathrm{L}$ & -34 & -84 & 2 & 59 & 6.47 \\
\hline Middle occipital gyrus & $\mathrm{R}$ & 30 & -80 & 10 & 53 & 7.53 \\
\hline Precentral gyrus & $\mathrm{L}$ & -54 & -2 & 46 & 19 & 5.83 \\
\hline Middle frontal gyrus & $\mathrm{L}$ & -24 & 8 & 40 & 4 & 4.22 \\
\hline Middle frontal gyrus & $\mathrm{R}$ & 30 & 20 & 40 & 10 & 5.05 \\
\hline Putamen & $\mathrm{L}$ & -24 & 2 & 4 & 131 & 6.56 \\
\hline Putamen & $\mathrm{R}$ & 24 & -4 & 4 & 36 & 5.22 \\
\hline \multicolumn{7}{|c|}{ Unpleasant Viewing Versus Neutral Viewing } \\
\hline Middle occipital gyrus & $\mathrm{L}$ & -38 & -84 & 8 & 123 & 5.80 \\
\hline Middle insula & $\mathrm{L}$ & -32 & -14 & 16 & 19 & 6.96 \\
\hline Amygdala & $\mathrm{L}$ & -26 & -6 & -14 & 133 & 2.33 \\
\hline Amygdala & $\mathrm{R}$ & 32 & -2 & -18 & 24 & 2.22 \\
\hline \multicolumn{7}{|c|}{ Unpleasant Detection Versus Neutral Detection } \\
\hline Inferior parietal gyrus & $\mathrm{L}$ & -40 & -40 & 52 & 31 & 5.53 \\
\hline Precentral gyrus & $\mathrm{L}$ & -26 & -20 & 68 & 51 & 6.27 \\
\hline Postcentral gyrus & $\mathrm{L}$ & -58 & -4 & 42 & 6 & 5.49 \\
\hline Superior frontal gyrus & $\mathrm{L}$ & -18 & -2 & 56 & 10 & 4.99 \\
\hline Midcingulate cortex & $\mathrm{L}$ & -4 & 8 & 46 & 9 & 4.44 \\
\hline Putamen & $\mathrm{L}$ & -18 & 4 & 12 & 11 & 5.43 \\
\hline
\end{tabular}

Note-All regions at $p<.001$, uncorrected, except the amygdala, at $p<.05 . \mathrm{L}=$ left, $\mathrm{R}=$ right; $x, y, z=$ MNI coordinates of the maximally active voxel; $t=$ maximum $t$ value.

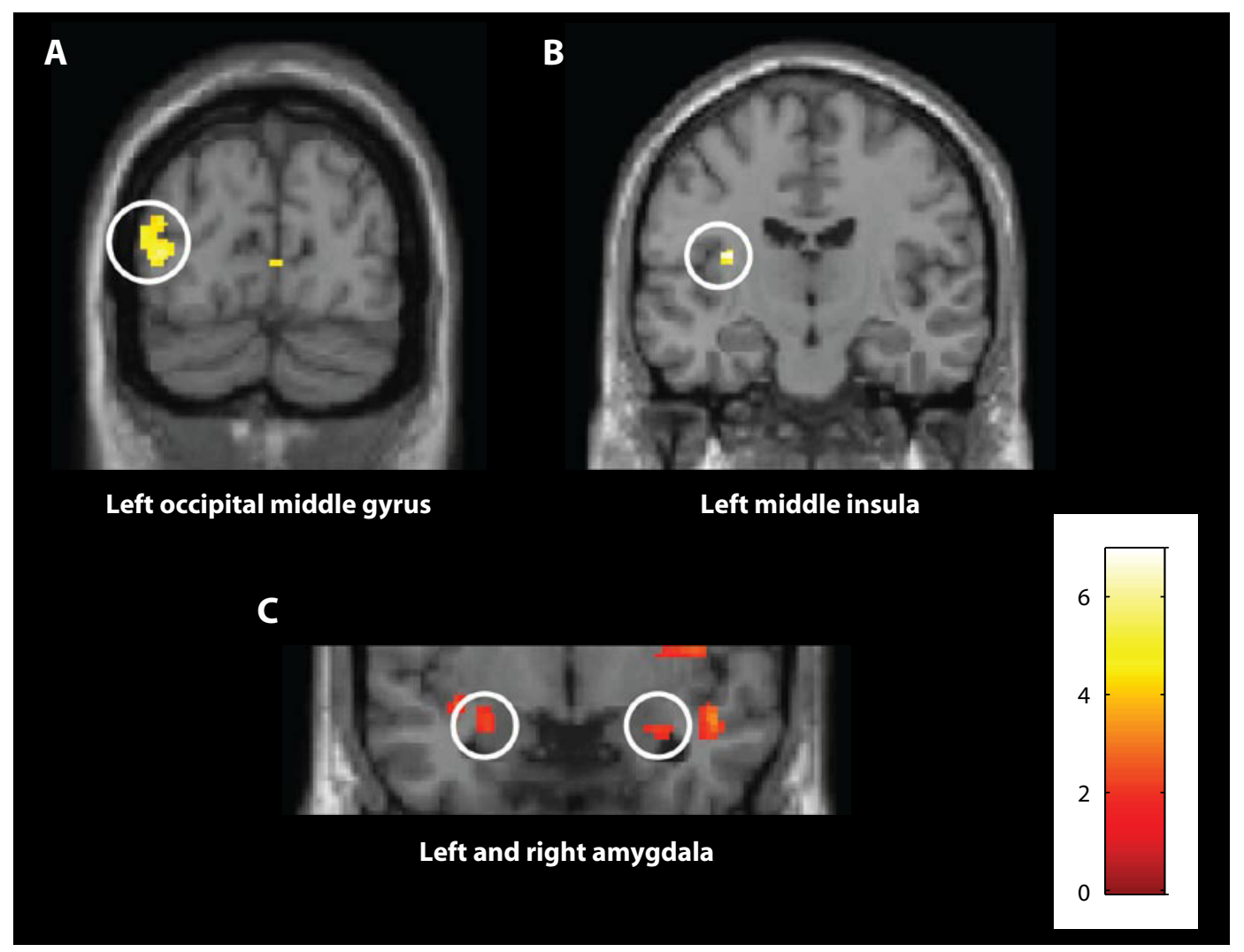

Figure 6. Voxelwise group maps illustrating the unpleasant-viewing versus neutral-viewing contrast. (A) Visual cortex (MNI coordinates: $x=-38, y=-84, z=8$ ). (B) Middle insula (MNI coordinates: $-32,-14,16$ ). (C) Amygdala left (MNI coordinates: $-26,-4,-14$ ) and right (MNI coordinates: 32, -4, -18). The gradient scale represents $z$ values. 


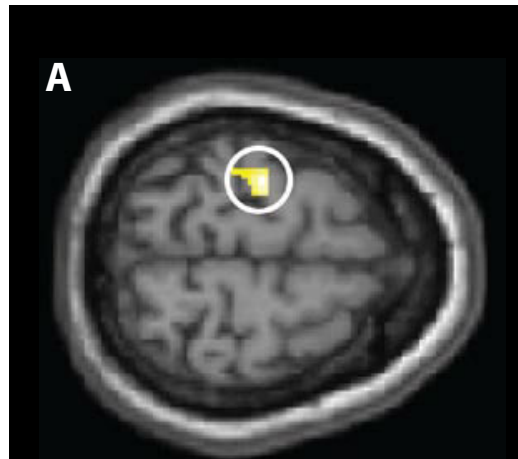

Left precentral gyrus

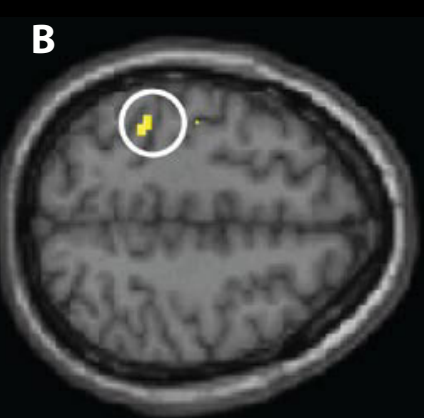

Inferior parietal gyrus

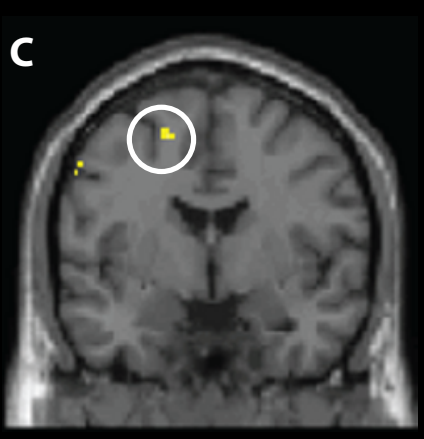

Left superior frontal gyrus

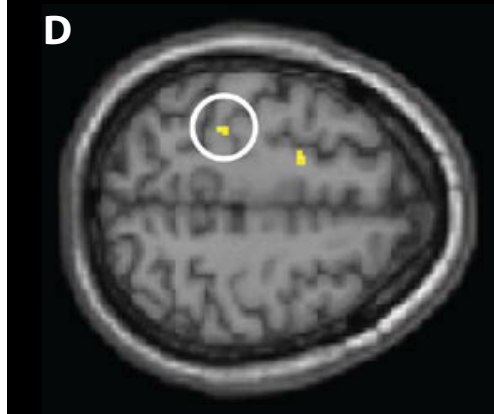

Left postcentral gyrus

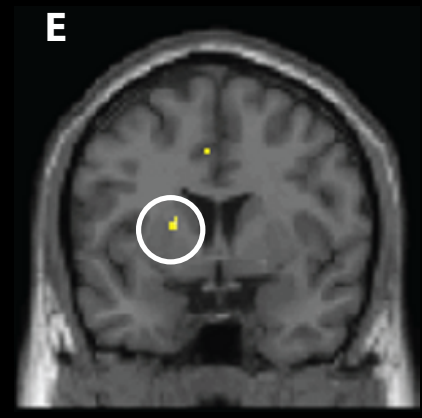

Left putamen

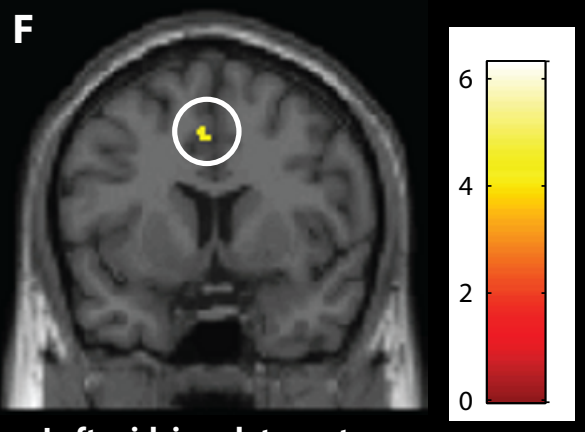

Left midcingulate cortex

Figure 7. Voxelwise group maps illustrating the unpleasant-detection versus neutral-detection contrast. See Table 1 for MNI coordinates of each cluster.

after the viewing of unpleasant pictures. Brain activity was modulated by the emotional context across most regions engaged by the target detection task. Interestingly, modulation of activity in the midcingulate cortex during target detection seemed to mirror the pattern of behavioral interference. Differential (unpleasant vs. neutral) evoked activity in this structure was relatively sustained during the detection phase and was correlated with the magnitude of the behavioral interference. Furthermore, emotional modulation in the midcingulate cortex during target detection was correlated with emotional modulation of midinsula activity during picture viewing. Taken together, our findings suggest that the cingulate cortex is an important site for the interaction between negatively valenced and motor information.

\section{Brain Areas Modulated by \\ Unpleasant Picture Viewing}

The unpleasant versus neutral viewing contrast revealed increased responses in visual cortex, amygdala, and middle insula. The ability of emotional stimuli to modulate ongoing visual processing is well documented, and all of the occipito-temporal cortex appears to be robustly modulated by stimulus valence (Bradley et al., 2003; Ishai, Pessoa, Bikle, \& Ungerleider, 2004; Lane, Chua, \& Dolan, 1999; Lang et al., 1998; Mourão-Miranda et al., 2003; Pessoa et al., 2002). Increased responses in the amygdala during unpleasant versus neutral picture viewing is, of course, consistent with a considerable body of data documenting how this structure is involved in affective processing (for a review, see Phan, Wager, Taylor, \& Liberzon, 2002); note, however, that amygdala activation was weak and was detected only at less stringent thresholds (see the Limitations of the Present Study section below).

Finally, the middle insula has been associated with a range of functions, including viscerosensation (Craig, 2003) and pain perception (Coghill, Sang, Maisog, \& Iadarola, 1999; Peyron, Laurent, \& García-Larrea, 2000). Indeed, it has recently been suggested that the middle insula integrates homeostatic representations with activity that is linked with emotionally salient information (Craig, 2009). In general, the insula may serve to monitor the ongoing internal emotional state of the organism and integrate sensory information with motivational salience to guide behavioral responses (Craig, 2009; Damasio, 1999).

\section{Midcingulate Cortex As a Pivotal Node of Emotional and Motor Interaction}

The unpleasant-detection versus neutral-detection contrast revealed an activation site in the midcingulate cortex. In addition, differential midcingulate activity appeared to be sustained during the target detection phase, a pattern similar to that observed behaviorally. These findings suggest that, in the present paradigm, the midcingulate may have integrated emotion and motor signals, an interpretation consistent with a growing body 
of work, as discussed below. Here, the focus of activation observed during the unpleasant-detection condition (as opposed to the neutral condition) was located at the aMCC/pMCC border (i.e., extending anteriorly to the aMCC), two subdivisions of the midcingulate cortex proposed by Vogt (for a review, see Vogt, 2005). Both subdivisions are important sites observed in pain studies (Strigo, Duncan, Boivin, \& Bushnell, 2003; Svensson, Minoshima, Beydoun, Morrow, \& Casey, 1997; Vogt, Berger, \& Derbyshire, 2003). Responses in the aMCC are enhanced by fear, too (Vogt et al., 2003). Vogt et al. (2003, p. 3142) suggested that the "aMCC coordinates fear and avoidance with skeletomotor activity through the rCMA [rostral cingulate motor areas].” More generally, the cingulate motor cortex receives widespread emotionrelated input, including signals from the orbitofrontal cortex and insula (Morecraft \& Van Hoesen, 1998). Indeed, the importance of the cingulate motor area as a pivotal point where emotion-related signals influence the voluntary motor system was also advanced by Morecraft and Van Hoesen (1998) (see also Paus, 2001).

Interestingly, in the present study, midinsula activity during the viewing of unpleasant pictures was positively correlated with midcingulate responses when the participants performed the target detection task within the same block. If we consider responses in the middle insula as an index of the emotional impact of picture viewing, the observed correlation suggests that such emotional impact might be predictive of the subsequent behavioral interference effect. We thus suggest that the middle insula might have been involved in mapping homeostatic and negatively valenced representations and that it conveyed such information to "motivational drive" representations in the midcingulate cortex and other cingulate sites (Craig, 2003, 2009; Critchley, Wiens, Rotshtein, Öhman, \& Dolan, 2004). Although tentative, this interpretation may be assessed further with network-related analysis techniques, such as dynamic causal modeling (Friston, Harrison, \& Penny, 2003), and may profit from larger sample sizes (see also Taylor, Seminowicz, \& Davis, 2009), for evidence of functional interactions between the middle insula and midcingulate cortex). It should be pointed out that the middle insula also exhibited activation during the target detection phase when a more lenient statistical threshold was adopted ( $p<.005$, uncorrected). Indeed, it appears that responses in this structure were also somewhat sustained following the picture-viewing phase and extended into the target detection phase (Figure 4). For evidence of sustained activation of the insula during blocked presentation of emotional stimuli, see also Wendt, Lotze, Weike, Hosten, and Hamm (2008).

Performing the target detection task during the unpleasant block engaged additional regions of the motor network, including the primary motor cortex and bilateral putamen. Because the cingulate motor cortex projects to these and other motor structures (Morecraft et al., 1997; Morecraft \& Van Hoesen, 1992), one may hypothesize that, in our experiment, the midcingulate conveyed affective information to other components of the voluntary motor system network during a negative context.
Interference effects induced by emotional stimuli have often been interpreted in terms of attention (Vuilleumier, 2005). Accordingly, an alternative explanation for the present behavioral effects of unpleasant pictures is that they captured attention, reducing the resources available for the subsequent target detection task. Interference effects observed for the very first target detection trial are indeed consistent with the timing associated with attentional processes, but attention likely does not account for the longer lasting interference effect described here. In general, selective attention effects are relatively fast and transient; for example, attentional dwell time is suggested to last on the order of $500 \mathrm{msec}$ or slightly longer (600$800 \mathrm{msec}$ ) (Müller, Teder-Sälejärvi, \& Hillyard, 1998). Thus, we favor the interpretation that the origin of this longer lasting interference effect is not attentional (at least in terms of a traditional attentional role) but is instead associated with the induction of an emotional state that is possibly linked to the activation of defensive responses, as outlined below.

\section{Midcingulate Cortex and the Implementation of Defensive Responses}

Our findings of the modulation of midcingulate responses by negatively valenced information during the target detection task also deserve to be discussed in the context of recent studies that indicate that this region is involved in the implementation of defensive responses. For instance, Morrison et al. (2007) asked participants to execute or suppress a motor response after viewing short animations depicting a noxious implement (e.g., a sharp knife) or an innocuous implement (e.g., a butter knife) striking a person's hand. The combination of the implement's noxiousness and whether it contacted the hand strongly affected RTs. Responses in the midcingulate cortex mirrored this behavioral interaction pattern, leading the authors to suggest that distinct areas within the cingulate gyrus, which were also engaged, may work together during unpleasant contexts to recognize the aversive nature of the event, mount an appropriate motor response, and modulate this response according to current task constraints. In this context, it should be noted that the unpleasant stimulus category employed here involved mutilation pictures, which may be among the most emotionally evocative (Bradley et al., 2001; Sarlo, Buodo, Poli, \& Palomba, 2005).

More direct evidence of the involvement of the cingulate cortex with the expression of defensive responses was reported in a study by Kalin, Shelton, Fox, Oakes, and Davidson (2005). In that study, freezing behavior was investigated in monkeys, and it was found that responses in the dorsal ACC were positively correlated with freezing duration in aversive contexts. Another study (Milad et al., 2007) addressed the role of the cingulate cortex in the expression of fear responses in humans. Increased activation, which included the midcingulate cortex by a conditioned fear stimulus, was reported, and these signals were positively correlated with increased fear responses (as indexed via skin conductance responses), suggesting that the cingulate cortex might be involved in the ex- 
pression of fear responses in general. Indeed, in a recent compilation of activation sites observed in conditioning studies (Engelmann, Damaraju, Padmala, \& Pessoa, 2009; Pessoa, 2009), we showed that cingulate sites, including the midcingulate cortex, are frequently engaged during the processing of aversive stimuli-an observation that, together with the findings of the present study and those reviewed here (see also Sommer, Hajak, Döhnel, Meinhardt, \& Müller, 2008), challenges the popular notion that more anterior and posterior portions of the anterior cingulate cortex are only specialized for affective and cognitive information, respectively (Bush, Luu, \& Posner, 2000). Taken together, these studies suggest that the cingulate cortex, including the midcingulate cortex observed in the present study, likely also has an important role in the implementation of defensive behaviors, which involve the integration of negatively valenced information and motor information.

In conclusion, we can hypothesize that, during the unpleasant block, the midcingulate cortex participated in the implementation of a defensive response, possibly a freezinglike response. Viewing an injured individual, such as was present in the mutilation pictures, may signal a potential life threat in the environment. Thus, it is possible that emitting a motor response that was unrelated to this defensive context, such as was done during the target detection task, involved increased activation in motor-related areas and produced behavioral interference. Evidence for the induction of a freezing-like response in humans by mutilation pictures has been reported by Azevedo et al. (2005). Posturographic and electrocardiographic recordings revealed immobility, rigidity, and bradycardia during the viewing of mutilation pictures (see also Facchinetti, Imbiriba, Azevedo, Vargas, \& Volchan, 2006).

In this context, an alternative hypothesis for the findings reported here is worth discussing. Specifically, the increased midcingulate activity during the unpleasant condition may have reflected response conflict. According to this idea, midcingulate responses may reflect the detection of the conflict between the action required by the imperative stimulus (i.e., a motor response) and an avoidance response, possibly freezing, evoked by the aversive context. Hence, increased midcingulate cortex signals might reflect the conflict generated by the coactivation of these two responses. It is well known that the anterior cingulate cortex (ACC) is engaged during the monitoring and/or detection of conflict (Barch, Braver, Sabb, \& Noll, 2000; Botvinick, Nystrom, Fissell, Carter, \& Cohen, 1999; Carter et al., 2000; Casey et al., 2000). It is noteworthy, however, that, according to Picard and Strick (2001), on average, the sites of conflict-related activations at the time were located $24 \mathrm{~mm}(S D=7)$ anterior to the level of the anterior commissure. It thus appears that the present activation site of the midcingulate cortex is posterior to those observed during conflict tasks (see Picard \& Strick, 2001).

\section{Limitations of the Present Study}

A limitation of the present study is the extent to which the present results generalize to other types of emotional stimuli. In the present study, as in our previous one (Pereira et al., 2006), we chose mutilation pictures because we intended to evoke strong aversive reactions. Naturally, a disadvantage of our design is that the results cannot be generalized to other classes of stimuli. We note, however, that in a previous study that used a very similar paradigm (Pereira et al., 2006), pleasant pictures of babies and of family interactions actually produced an acceleration of RT when stimuli were presented in a blocked fashion, as was done in the present study. These findings lend credence to the notion that the present results are valence specific, but this suggestion is tentative at present.

A second important limitation of the present study is that some of the results concerning the amygdala - a critical structure in the processing of affective stimuli-were observed only at lenient statistical thresholds. Although we do not advocate the use of different statistical thresholds in general, in the present study, we considered this strategy acceptable, given that a vast literature has identified amygdala responses in similar conditions to those probed here. In other words, given the extant literature, it is unlikely that the observed responses in the amygdala reflect a gross Type I statistical error.

\section{Implications for Affective Disorders}

Understanding how negative emotion impairs behavior and the underlying neural circuits has important implications for affective disorders-especially depression, which is characterized by persistent negative mood and selective cognitive and behavioral disturbances. For example, depressed patients show a failure to inhibit negative information (Goeleven, De Raedt, Baert, \& Koster, 2006), which might be related to decreased behavioral adjustments. Our findings revealed that the midcingulate cortex was more strongly engaged during the unpleasant context, and activation of this region has been shown to be involved in the ability to adaptively regulate freezing as a function of a changing context (Kalin et al., 2005). Interestingly, it has recently been suggested that the anterior midcingulate cortex might play a protective role in the pathogenesis of depression; individuals with reduced gray-matter volume and functional activation of this region might be predisposed to more severe depression symptoms (Chen et al., 2007). Given that the midcingulate cortex might be involved in adaptively regulating freezing, it would potentially be valuable to test whether these patients exhibit abnormalities in the engagement of motor circuits during emotion-laden contexts, such as those studied here. It is conceivable, for instance, that depressed patients exhibit an enhanced freezing-like state, as compared with nonclinical individuals.

Given the high comorbidity between depression and anxiety disorders, our findings may also be of interest in the context of the latter condition. Recent studies have investigated the role of the ACC in generalized anxiety disorder and reported that heightened activity in this region may be "adaptive" (Nitschke et al., 2009; Whalen et al., 2008). For instance, patients with increased ACC activity exhibited greater reductions in anxiety and worry symptoms after pharmacological treatment. As suggested 
by Nitschke et al., increased activity in the ACC may be an indication of unimpaired top-down regulation in patients with more favorable outcomes. It is important to bear in mind, however, that the cingulate cortex findings observed here were posterior to the ACC, although the two regions are strongly interconnected (Morecraft \& Van Hoesen, 1998).

\section{CONCLUDING REMARKS}

As has been shown previously, the sight of mutilation pictures leads to strong physiological reactions and evaluative reports of high arousal and unpleasantness (Bradley et al., 2001). We conjecture that, in the present study, these alterations of body physiology were represented by the middle insula and that these negatively valenced signals were complemented by a parallel motivational drive for action triggered by the midcingulate cortex. An appropriate response in this context is the installation of a defense pattern - probably freezing, as discussed above. In our task, the immobility aspect of the defense pattern probably competed with the requirement to emit a motor response, possibly resulting in an increased recruitment of the motor circuits necessary to execute the task. Our results suggest that, in addition to privileging capture of attention and consumption of processing resources (Pessoa et al., 2002; Vuilleumier, 2005), viewing unpleasant stimuli also modulates output systems. Thus, emotional modulation by negative stimuli-and, particularly, behavioral interference - might be partially a reflection of the prompting of defensive responses and how they are related to ongoing behaviors.

\section{AUTHOR NOTE}

We thank the anonymous reviewers for their careful feedback, and Rita de Cássia Soares Alves for collecting the behavioral data of participants who performed the task outside the scanner. This work was supported by funds from federal and state Brazilian research agencies (CNPq, CAPES, PROCAD, PRONEX/FAPERJ, FAPERJ, and IBN-NET FINEP) and was supported in part by National Institute of Mental Health Grant MH071589 to L.P. Correspondence concerning this article should be addressed to L. Pessoa, Department of Psychological and Brain Sciences, Indiana University, 1101 E. 10th Street, Bloomington, IN 47405 (e-mail: lpessoa@indiana.edu).

\section{REFERENCES}

Anderson, A. K., \& Phelps, E. A. (2001). Lesions of the human amygdala impair enhanced perception of emotionally salient events. Nature, 411, 305-309.

Azevedo, T. M., Volchan, E., Imbiriba, L. A., Rodrigues, E. C., Oliveira, J. M., Oliveira, L. F., ET AL. (2005). A freezing-like posture to pictures of mutilation. Psychophysiology, 42, 255-260.

Barch, D. M., Braver, T. S., SabB, F. W., \& Noll, D. C. (2000). Anterior cingulate and the monitoring of response conflict: Evidence from an fMRI study of overt verb generation. Journal of Cognitive Neuroscience, 12, 298-309.

Baumgartner, T., Willi, M., \& Jäncke, L. (2007). Modulation of corticospinal activity by strong emotions evoked by pictures and classical music: A transcranial magnetic stimulation study. NeuroReport, 18, 261-265.

BECKER, M.W. (2009). Panic search: Fear produces efficient visual search for nonthreatening objects. Psychological Science, 20, 435-437.

Bocanegra, B. R., \& ZeElenberg, R. (2009). Emotion improves and impairs early vision. Psychological Science, 20, 707-713.
Botvinick, M., Nystrom, L. E., Fissell, K., Carter, C. S., \& Cohen, J. D. (1999). Conflict monitoring versus selection-for-action in anterior cingulate cortex. Nature, 402, 179-181.

Bradley, M. M., Codispoti, M., Cuthbert, B. N., \& Lang, P. J. (2001). Emotion and motivation I: Defensive and appetitive reactions in picture processing. Emotion, 1, 276-298.

Bradley, M. M., Cuthbert, B. N., \& Lang, P. J. (1996). Picture media and emotion: Effects of a sustained affective context. Psychophysiology, 33, 662-670.

Bradley, M. M., \& Lang, P. J. (1994). Measuring emotion: The SelfAssessment Manikin and the semantic differential. Journal of Behavior Therapy \& Experimental Psychiatry, 25, 49-59.

Bradley, M. M., Sabatinelli, D., Lang, P. J., Fitzsimmons, J. R., KING, W., \& DesaI, P. (2003). Activation of the visual cortex in motivated attention. Behavioral Neuroscience, 117, 369-380.

Bush, G., LuU, P., \& Posner, M. I. (2000). Cognitive and emotional influences in anterior cingulate cortex. Trends in Cognitive Sciences, 4, 215-222.

Butler, T., Pan, H., Tuescher, O., Engelien, A., Goldstein, M., EPSTEIN, J., ET AL. (2007). Human fear-related motor neurocircuitry. Neuroscience, 150, 1-7.

Carter, C. S., MacDonald, A. M., Botvinick, M., Ross, L. L., Stenger, V. A., Noll, D., \& Cohen, J. D. (2000). Parsing executive processes: Strategic vs. evaluative functions of the anterior cingulate cortex. Proceedings of the National Academy of Sciences, 97, 19441948.

Casey, B. J., Thomas, K. M., Welsh, T. F., Badgaiyan, R. D., Eccard, C. H., Jennings, J. R., \& Crone, E. A. (2000). Dissociation of response conflict, attentional selection, and expectancy with functional magnetic resonance imaging. Proceedings of the National Academy of Sciences, 97, 8728-8733.

Chen, C.-H., Ridler, K., Suckling, J., Williams, S., Fu, C. H. Y., Merlo-Pich, E., \& Bullmore, E. (2007). Brain imaging correlates of depressive symptom severity and predictors of symptom improvement after antidepressant treatment. Biological Psychiatry, 62, 407-414.

Coghill, R. C., Sang, C. N., Maisog, J. M., \& Iadarola, M. J. (1999). Pain intensity processing within the human brain: A bilateral, distributed mechanism. Journal of Neurophysiology, 82, 1934-1943.

Craig, A. D. (2003). Interoception: The sense of the physiological condition of the body. Current Opinion in Neurobiology, 13, 500-505.

Craig, A. D. (2009). How do you feel-now? The anterior insula and human awareness. Nature Reviews Neuroscience, 10, 59-70.

Critchley, H. D., Wiens, S., Rotshtein, P., Öhman, A., \& Dolan, R. J. (2004). Neural systems supporting interoceptive awareness. $\mathrm{Na}$ ture Neuroscience, 7, 189-195.

Damasio, A. (1999). The feelings of what happens: Body and emotion in the making of consciousness (1st ed.). New York: Harcourt Brace.

DARWIN, C. (1872). The expression of the emotions in man and animals. London: John Murray.

Engelmann, J. B., Damaraju, E., Padmala, S., \& Pessoa, L. (2009). Combined effects of attention and motivation on visual task performance: Transient and sustained motivational effects. Frontiers in Human Neuroscience, 3(Art. 4), 1-17.

Erthal, F. S., de Oliveira, L., Mocaiber, I., Pereira, M. G., Machado-Pinheiro, W., Volchan, E., \& Pessoa, L. (2005). Loaddependent modulation of affective picture processing. Cognitive, Affective, \& Behavioral Neuroscience, 5, 388-395.

Facchinetti, L. D., Imbiriba, L. A., Azevedo, T. M., Vargas, C. D., \& Volchan, E. (2006). Postural modulation induced by pictures depicting prosocial or dangerous contexts. Neuroscience Letters, 410, 52-56.

Friston, K. J., Harrison, L., \& Penny, W. (2003). Dynamic causal modelling. NeuroImage, 19, 1273-1302.

Friston, K. J., Holmes, A. P., Worsley, K. J., Poline, J.-P., Frith, C. D., \& FrackowiaK, R. S. J. (1994). Statistical parametric maps in functional imaging: A general linear approach. Human Brain Mapping, 2, 189-210.

Goeleven, E., De Raedt, R., Baert, S., \& Koster, E. H. W. (2006). Deficient inhibition of emotional information in depression. Journal of Affective Disorders, 93, 149-157.

Graziano, M. S. A., \& CooKe, D. F. (2006). Parieto-frontal interactions, personal space, and defensive behavior. Neuropsychologia, 44, 2621 2635. 
Hajcak, G., Molnar, C., George, M. S., Bolger, K., Koola, J., \& NAHAS, Z. (2007). Emotion facilitates action: A transcranial magnetic stimulation study of motor cortex excitability during picture viewing. Psychophysiology, 44, 91-97.

Hartikainen, K. M., Ogawa, K. H., \& Knight, R. T. (2000). Transient interference of right hemispheric function due to automatic emotional processing. Neuropsychologia, 38, 1576-1580.

Ishai, A., Pessoa, L., Bikle, P. C., \& Ungerleider, L. G. (2004). Repetition suppression of faces is modulated by emotion. Proceedings of the National Academy of Sciences, 101, 9827-9832.

Kalin, N. H., Shelton, S. E., Fox, A. S., Oakes, T. R., \& Davidson, R. J. (2005). Brain regions associated with the expression and contextual regulation of anxiety in primates. Biological Psychiatry, 58, 796-804.

Koski, L., \& PAUS, T. (2000). Functional connectivity of the anterior cingulate cortex within the human frontal lobe: A brain-mapping metaanalysis. Experimental Brain Research, 133, 55-65.

Koster, E. H. W., Crombez, G., Verschuere, B., \& De Houwer, J. (2004). Selective attention to threat in the dot probe paradigm: Differentiating vigilance and difficulty to disengage. Behaviour Research \& Therapy, 42, 1183-1192.

Kriegeskorte, N., Simmons, W. K., Bellgowan, P. S. F., \& BaKer, C. I. (2009). Circular analysis in systems neuroscience: The dangers of double dipping. Nature Neuroscience, 12, 535-540.

Lane, R. D., Chua, P. M.-L., \& Dolan, R. J. (1999). Common effects of emotional valence, arousal and attention on neural activation during visual processing of pictures. Neuropsychologia, 37, 989-997.

Lang, P. J., BradLey, M. M., \& CuthBert, B. N. (1997). International affective picture system (IAPS): Technical manual and affective ratings. Gainesville, FL: NIMH Center for the Study of Emotion and Attention.

Lang, P. J., Bradley, M. M., Fitzsimmons, J. R., Cuthbert, B. N., ScotT, J. D., Moulder, B., \& NANGIA, V. (1998). Emotional arousal and activation of the visual cortex: An fMRI analysis. Psychophysiology, 35, 199-210.

Milad, M. R., Quirk, G. J., Pitman, R. K., OrR, S. P., Fischl, B., \& RaUCH, S. L. (2007). A role for the human dorsal anterior cingulate cortex in fear expression. Biological Psychiatry, 62, 1191-1194.

Morecraft, R. J., Louie, J. L., Schroeder, C. M., \& Avramov, K. (1997). Segregated parallel inputs to the brachial spinal cord from the cingulate motor cortex in the monkey. NeuroReport, 8, 3933-3938.

Morecraft, R. J., \& Van Hoesen, G. W. (1992). Cingulate input to the primary and supplementary motor cortices in the rhesus monkey: Evidence for somatotopy in areas $24 \mathrm{c}$ and $23 \mathrm{c}$. Journal of Comparative Neurology, 322, 471-489.

Morecraft, R. J., \& Van Hoesen, G. W. (1998). Convergence of limbic input to the cingulate motor cortex in the rhesus monkey. Brain Research Bulletin, 45, 209-232.

Morrison, I., Peelen, M. V., \& Downing, P. E. (2007). The sight of others' pain modulates motor processing in human cingulate cortex. Cerebral Cortex, 17, 2214-2222.

Mourão-Miranda, J., Volchan, E., Moll, J., De Oliveira-Souza, R., Oliveira, L., Bramati, I., ET AL. (2003). Contributions of stimulus valence and arousal to visual activation during emotional perception. NeuroImage, 20, 1955-1963.

Müller, M. M., Teder-Sälejärvi, W., \& Hillyard, S. A. (1998). The time course of cortical facilitation during cued shifts of spatial attention. Nature Neuroscience, 1, 631-634.

Nitschke, J. B., Sarinopoulos, I., OAthes, D. J., Johnstone, T., Whalen, P. J., Davidson, R. J., \& Kalin, N. H. (2009). Anticipatory activation in the amygdala and anterior cingulate in generalized anxiety disorder and prediction of treatment response. American Journal of Psychiatry, 166, 302-310.

Oliveri, M., Babiloni, C., Filippi, M. M., Caltagirone, C., BabiLONI, F., CiCINELLI, P., ET AL. (2003). Influence of the supplementary motor area on primary motor cortex excitability during movements triggered by neutral or emotionally unpleasant visual cues. Experimental Brain Research, 149, 214-221.

Paus, T. (2001). Primate anterior cingulate cortex: Where motor control, drive and cognition interface. Nature Reviews Neuroscience, 2, 417-424.
Pereira, M. G., Volchan, E., De Souza, G. G. L., Oliveira, L., CamPagnoli, R. R., Pinheiro, W. M., \& Pessoa, L. (2006). Sustained and transient modulation of performance induced by emotional picture viewing. Emotion, 6, 622-634.

Pereira, M. G., Volchan, E., Oliveira, L., Machado-Pinheiro, W., Rodrigues, J. A., Nepomuceno, F. V. P., \& Pessoa, L. (2004). Behavioral modulation by mutilation pictures in women. Brazilian Journal of Medical \& Biological Research, 37, 353-362.

PessoA, L. (2009). How do emotion and motivation direct executive control? Trends in Cognitive Sciences, 13, 160-166.

Pessoa, L., Kastner, S., \& Ungerleider, L. G. (2002). Attentional control of the processing of neutral and emotional stimuli. Cognitive Brain Research, 15, 31-45.

Peyron, R., Laurent, B., \& García-Larrea, L. (2000). Functional imaging of brain responses to pain: A review and meta-analysis. $\mathrm{Neu}$ rophysiologie Clinique, 30, 263-288.

Phan, K. L., Wager, T., Taylor, S. F., \& Liberzon, I. (2002). Functional neuroanatomy of emotion: A meta-analysis of emotion activation studies in PET and fMRI. NeuroImage, 16, 331-348.

Phelps, E. A., Ling, S., \& Carrasco, M. (2006). Emotion facilitates perception and potentiates the perceptual benefits of attention. Psychological Science, 17, 292-299.

Phelps, E. A., O’Connor, K. J., Gatenby, J. C., Gore, J. C., GrilLON, C., \& DAvis, M. (2001). Activation of the left amygdala to a cognitive representation of fear. Nature Neuroscience, 4, 437-441.

PICARD, N., \& STRICK, P. L. (2001). Imaging the premotor areas. Current Opinion in Neurobiology, 11, 663-672.

Sarlo, M., Buodo, G., Poli, S., \& Palomba, D. (2005). Changes in EEG alpha power to different disgust elicitors: The specificity of mutilations. Neuroscience Letters, 382, 291-296.

Sommer, M., HajaK, G., Döhnel, K., Meinhardt, J., \& Müller, J. L. (2008). Emotion-dependent modulation of interference processes: An fMRI study. Acta Neurobiologiae Experimentalis, 68, 193-203.

Strigo, I. A., Duncan, G. H., Boivin, M., \& Bushnell, M. C. (2003). Differentiation of visceral and cutaneous pain in the human brain. Journal of Neurophysiology, 89, 3294-3303.

Svensson, P., Minoshima, S., Beydoun, A., Morrow, T. J., \& Casey, K. L. (1997). Cerebral processing of acute skin and muscle pain in humans. Journal of Neurophysiology, 78, 450-460.

TAYlOR, K. S., SEminowicz, D. A., \& Davis, K. D. (2009). Two systems of resting state connectivity between the insula and cingulate cortex. Human Brain Mapping, 30, 2731-2745.

Tipples, J., \& Sharma, D. (2000). Orienting to exogenous cues and attentional bias to affective pictures reflect separate processes. British Journal of Psychology, 91, 87-97.

VogT, B. A. (2005). Pain and emotion interactions in subregions of the cingulate gyrus. Nature Reviews Neuroscience, 6, 533-544.

Vogt, B. A., Berger, G. R., \& Derbyshire, S. W. G. (2003). Structural and functional dichotomy of human midcingulate cortex. European Journal of Neuroscience, 18, 3134-3144.

Vuilleumier, P. (2005). How brains beware: Neural mechanisms of emotional attention. Trends in Cognitive Sciences, 9, 585-594.

Vul, E., Harris, C., Winkielman, P., \& Pashler, H. (2009). Puzzlingly high correlations in fMRI studies of emotion, personality, and social cognition. Perspectives on Psychological Science, 4, 274-290.

Wendt, J., Lotze, M., Weike, A. I., Hosten, N., \& Hamm, A. O. (2008). Brain activation and defensive response mobilization during sustained exposure to phobia-related and other affective pictures in spider phobia. Psychophysiology, 45, 205-215.

Whalen, P. J., Johnstone, T., Somerville, L. H., Nitschke, J. B., Polis, S., AleXAnder, A. L., ET AL. (2008). A functional magnetic resonance imaging predictor of treatment response to venlafaxine in generalized anxiety disorder. Biological Psychiatry, 63, 858-863.

Worsley, K. J., Liao, C. H., Aston, J., Petre, V., Duncan, G. H., Morales, F., \& Evans, A. C. (2002). A general statistical analysis for fMRI data. Neurolmage, 15, 1-15.

(Manuscript received April 28, 2009; revision accepted for publication October 30, 2009.) 